\title{
Intranasal Delivery of Obidoxime to the Brain Prevents Mortality and CNS Damage from Organophosphate Poisoning
}

Jishnu K.S. Krishnan ${ }^{1}$, Peethambaran Arun ${ }^{1}$, Abhilash P. Appu ${ }^{1}$, Nivetha Vijayakumar ${ }^{1}$, Taíza H. Figueiredo ${ }^{1}$, Maria F. M. Braga ${ }^{1}$, Sudikshya Baskota ${ }^{1}$, Cara H. Olsen ${ }^{2}$, Natalia Farkas ${ }^{3}$, John Dagata ${ }^{3}$, William H. Frey $\mathrm{II}^{4}$, John R. Moffett ${ }^{1}$, and Aryan M. A. Namboodiri ${ }^{1 *}$

${ }^{1}$ Department of Anatomy, Physiology and Genetics, and Neurosciences Program, USUHS, Bethesda, MD

${ }^{2}$ Biostatistics Consulting Center, USUHS Bethesda, MD

${ }^{3}$ Section on Metrology, NIST, Gaithersburg, MD

${ }^{4}$ Center for Memory \& Aging, Regions Hospital, Health Partners Institute for Education and Research, St. Paul, MN

*corresponding author; Aryan M. A. Namboodiri, Department of Anatomy, Physiology and Genetics, and Neurosciences Program, USUHS, Bethesda, MD, 20814, phone; 301-295-3525, email; anamboodiri@usuhs.edu .

Running title: Intranasal obidoxime for OP poisoning

Abstract: Intranasal delivery is an emerging method for bypassing the blood brain barrier (BBB) and targeting therapeutics to the CNS. Oximes are used to counteract the effects of organophosphate poisoning, but they do not readily cross the BBB. Therefore, they cannot effectively counteract the central neuropathologies caused by cholinergic over-activation when administered peripherally. For these reasons we examined intranasal administration of oximes in an animal model of severe organophosphate poisoning to determine their effectiveness in reducing mortality and seizure-induced neuronal degeneration. Using the paraoxon model of organophosphate poisoning, we administered the standard treatment (intramuscular pralidoxime plus atropine sulphate) to all animals and then compared the effectiveness of intranasal application of obidoxime (OBD) to saline in the control groups. Intranasally administered OBD was effective in partially reducing paraoxon-induced acetylcholinesterase inhibition in the brain and substantially reduced seizure severity and duration. Further, intranasal OBD completely prevented mortality, which was $41 \%$ in the animals given standard treatment plus intranasal saline. Fluoro-Jade-B staining revealed extensive neuronal degeneration in the surviving salinetreated animals 24 hours after paraoxon administration, whereas no detectable degenerating neurons were observed in any of the animals given intranasal OBD $30 \mathrm{~min}$ before or 5 min after paraoxon administration. These findings demonstrate that intranasally administered oximes bypass the BBB more effectively than those administered peripherally and provide an effective method for protecting the brain from organophosphates. The addition of intranasally administered oximes to the current treatment regimen for organophosphate poisoning would improve efficacy, reducing both brain damage and mortality.

Keywords: acetylcholinesterase, excitotoxicity, Fluoro-Jade, neuropathology, Racine scale, status epilepticus

Abbreviations: 2-PAM = pralidoxime, $\mathrm{AChE}=$ acetylcholinesterase, $\mathrm{BBB}=$ blood brain barrier, $\mathrm{CTA}=$ chemical threat agent, FJB $=$ Fluoro-Jade B, OBD = obidoxime, SE status epilepticus 


\section{Introduction}

Organophosphate poisoning is a significant world health problem, claiming thousands of lives per year through intentional and unintentional pesticide exposure (Gunnell et al., 2007). Military and industrial organophosphates are also potential chemical threat agents (CTA) that can pose risks for both military personnel and civilian populations (Jett, 2007). Organophosphate based CTA exert their toxic effects by inhibiting acetylcholinesterase (AChE) leading to the accumulation of acetylcholine at synaptic and neuromuscular junctions. Excessive cholinergic activation in acute CTA poisoning causes prolonged seizures and status epilepticus (SE), excitotoxic brain injury, and can lead to death from cardiorespiratory failure. Treatment for acute organophosphate poisoning includes combinations of oximes to reactivate the inhibited AChE and atropine sulphate to antagonize the action of excess acetylcholine released at muscarinic receptors. In order to prevent seizure-associated CNS neuropathologies, anticonvulsants such as diazepam or midazolam are administered to potentiate the action of the inhibitory neurotransmitter $\gamma$-amino butyric acid. A major limitation of this therapeutic strategy is that oximes are hydrophilic and do not cross the BBB sufficiently to protect the CNS from excitotoxic damage when administered peripherally.

Intranasal drug administration permits rapid and effective targeting of therapeutics to the CNS (Thorne et al., 2004). Pralidoxime (2-PAM) and obidoxime (OBD) are oximes that have been used to treat organophosphate poisoning. Poor efficacy has been reported with 2-PAM in treating organophosphate poisoning patients (Buckley et al., 2011; Eddleston et al., 2009; Peter et al., 2006; Wille et al., 2013). OBD, while showing somewhat better efficacy than 2-PAM, is not able to reactivate AChE sufficiently to protect the brain from organophosphate poisoning (Eyer et al., 2009; Thiermann et al., 1997). This may be due in part to the fact that 2-PAM and OBD are administered intramuscularly or intravenously, and do not cross the BBB sufficiently to reach the CNS in concentrations that can effectively reverse central AChE inactivation. In order to reduce the acute neuronal damage and chronic neuropathological outcomes caused by CTAs (McDonough, Jr. and Shih, 1997), targeting of oximes to the CNS via the intranasal route could prove an effective strategy.

\section{Materials and Methods}

All animal experiments were conducted following the NIH Guidelines for the Care and Use of Laboratory Animals, and the animal protocol was approved by the animal care and use committee (IACUC) of the Uniformed Services University of the Health Sciences, Bethesda, MD. Adult male rats Sprague-Dawley rats (200 to $400 \mathrm{gm}$ ) were used for all studies (Taconic Biosciences, NY). Animals were housed individually in an environmentally controlled room (20$23^{\circ} \mathrm{C}$, 44\% humidity, 12 hour light/dark cycle, 350-400 lux, lights on at 6:00 am), with food (Teklad Global 18\% protein \#2018 rodent diet; Harlan Laboratories, IN) and water available continuously. Animal handling was minimized to reduce animal stress.

\subsection{Uptake of 2-PAM into the brain}

Our initial efforts were focused on nanotechnology based methods to bypass the BBB. Initial studies using chitosan-based nanoemulsion methods were unsuccessful due to insufficient 2-PAM incorporation into the emulsions, most likely because of the highly ionic nature of 2- 
PAM. Subsequently efforts using Chitosan-based nanoparticles prepared by an ionic gelation method appeared promising initially in view of the drug loading capacity and ease of preparation (see Supplemental Materials). However, the highly viscous nature of the final preparation prevented its use in intranasal brain delivery with the intranasal delivery device. Subsequently we found that highly concentrated aqueous solutions of 2-PAM $(0.5-1.0 \mathrm{~g} / \mathrm{ml})$ were effective for intranasal brain delivery. For most bioavailability studies we used 2-PAM as our model oxime. We custom synthesized radiolabeled $\left[{ }^{3} \mathrm{H}\right]-2-P A M$ with high specific activity $(20 \mathrm{Ci} / \mathrm{mmole}$; American Radiolabeled Chemicals, Inc., MO) using a published method (Balan et al., 1993). Samples were prepared by mixing $10-100 \mu \mathrm{Ci}$ of $\left[{ }^{3} \mathrm{H}\right]-2-\mathrm{PAM}$ with $100 \mu \mathrm{g}-30 \mathrm{mg}$ of unlabeled 2-PAM in normal saline. Adult male Sprague-Dawley rats were anesthetized with 5\% isoflurane (3 min) and maintained on $2 \%$ isoflurane until completion of the intranasal application procedure. Rats were kept in the supine position for optimal delivery of 2-PAM to the olfactory epithelium. 2-PAM (100 $\mu \mathrm{g}$ to $30 \mathrm{mg}$ ) in 10 to $30 \mu \mathrm{l}$ sterile saline (bilaterally, $5 \mu \mathrm{l} / \mathrm{nostril} / \mathrm{dose}$ with an interval of 3 min between the 3 dosages) was administered using an intranasal drug delivery device (Precision Olfactory Delivery (POD) device; Impel NeuroPharma). The animals were euthanized after different time intervals $(2.5 \mathrm{~min}$ to 1 hour). Except where noted in one experiment, animals were perfused transcardially with 100 to $150 \mathrm{ml}$ of phosphate buffered saline (PBS) to remove all $\left[{ }^{3} \mathrm{H}\right]-2-\mathrm{PAM}$ associated with the blood supply. Brains, upper spinal cords and trigeminal ganglia were rapidly removed and the brains were dissected into 6 different regions. All samples were rapidly frozen on dry ice. The $\left[{ }^{3} \mathrm{H}\right]-2-P A M$ was extracted in methanol and radioactivity was determined by liquid scintillation counting. The chloride forms of 2-PAM and OBD, and other reagents not indicated otherwise, were from Sigma Aldrich.

\subsection{HPLC analysis of $O B D$ concentrations in the brain after intranasal administration}

Ion pair chromatography-diode array detection (IPC-DAD) HPLC analysis of OBD concentrations in the brain were analyzed as follows. Aliquots $(200 \mu \mathrm{l})$ of homogenates from 7 different brain regions from rats administered saline or OBD intranasally were added to 9 volumes of methanol and centrifuged $(10,000 \mathrm{x}$ g/10min) to remove proteins. Supernatants were lyophilized and $100 \mu \mathrm{l}$ of HPLC buffer was added to the remaining pellet. Samples were analyzed with and without OBD standard to verify the identity of the sample peaks via comigration. Aliquots (40ul) were mixed with $10 \mu \mathrm{l}$ of standard OBD or $10 \mu \mathrm{l}$ HPLC buffer and were vortex mixed. Aliquots $(20 \mu \mathrm{l})$ were injected to a $\mathrm{C} 18$ reverse phase column (Thermo Scientific, $150 \mathrm{~mm} \times 4.6 \mathrm{~mm}$ ) using an Agilent Technologies 1200 Series HPLC system at $22^{\circ} \mathrm{C}$ with a flow rate of $1 \mathrm{ml} /$ minute. The mobile phase consisted of $20 \%$ acetonitrile, $5 \mathrm{mM}$ tetramethylammonium chloride and $5 \mathrm{mM}$ sodium octanesulfonate in phosphate buffer with the $\mathrm{pH}$ adjusted to 2.3. Peaks were detected via UV-absorbance at $296 \mathrm{~nm}$ using a diode array detector (Zdarova et al., 2010).

\subsection{Paraoxon administration and behavioral studies}

The experimental procedures for the OBD efficacy studies are outlined in Figure 1. Two sets of experiments were performed 1) seizure severity and neuropathology studies and 2) AChE activity studies. For both sets of experiments the treatment groups included rats treated intranasally with either OBD or saline either 30 minutes before or 5 minutes after paraoxon administration. In the pretreatment studies where OBD or saline were administered intranasally 30 min prior to paraoxon, 2-PAM and atropine, adult male Sprague-Dawley rats were anesthetized with $5 \%$ isoflurane for $4 \mathrm{~min}$ and maintained on $2 \%$ isoflurane $(5-6 \mathrm{~min})$ until 
completion of the intranasal administration procedure. In the post treatment studies where OBD or saline were administered intranasally $5 \mathrm{~min}$ after paraoxon, 2-PAM and atropine, adult male Sprague-Dawley rats were anesthetized with $2 \%$ isoflurane for 2 min after paraoxon administration, which was increased to $5 \%$ for $1 \mathrm{~min}$ to provide sufficient anesthesia for handling during intranasal dosing. For the AChE activity assays, the same procedures were used, where adult male Sprague-Dawley rats were administered OBD or saline intranasally either 30 min before or $5 \mathrm{~min}$ after paraoxon, 2-PAM and atropine. After one hour rats were deeply anesthetized with pentobarbital $(100 \mathrm{mg} / \mathrm{kg})$, brains were collected and dissected on ice into 7 different regions for $\mathrm{AChE}$ activity assays. Brain region samples were frozen at $-80{ }^{\circ} \mathrm{C}$ until assayed.

Paraoxon solutions were prepared fresh by adding $10 \mu \mathrm{l}$ stock solution $(1.274 \mathrm{~g} / \mathrm{mL})$ to 3 $\mathrm{ml}$ of ice cold PBS in a glass vial and mixing thoroughly. A fatal dose of paraoxon $(4 \mathrm{mg} / \mathrm{kg}$ (Deshpande et al., 2014)) was administered subcutaneously followed by intramuscular atropine sulphate $(2 \mathrm{mg} / \mathrm{kg})$ and intramuscular 2-PAM $(25 \mathrm{mg} / \mathrm{kg})$. Animals were treated intranasally with either OBD (38 mg dissolved in $100 \mu \mathrm{l}$ sterile water), or with sterile saline. For each rat, four intranasal POD device applicator tips were preloaded with OBD or saline (two tips with $30 \mu 1$, and two with $20 \mu \mathrm{l}$ ). The solutions were administered bilaterally with a 5 to 10 second interval between doses to each nostril, first administering the $30 \mu$ laliquots, immediately followed by the $20 \mu \mathrm{l}$ aliquots, for a total of $50 \mu \mathrm{l}$ of solution delivered to each nostril (Figure 1). Mild nose bleeding was observed in some rats and the bleeding was more common in rats given OBD than those given saline (8.6\% and $5.7 \%$ of rats respectively). Immediately after paraoxon, rats were observed independently by two trained researchers for signs of seizure onset, and continuously rated for seizure severity according to a modified Racine Scale: Stage 0, no behavioral response; Stage 1, behavioral arrest, orofacial movements, chewing; Stage 2, head nodding/myoclonus; Stage 3, unilateral/bilateral forelimb clonus without rearing, straub tail, extended body posture; Stage 4, bilateral forelimb clonus plus rearing; Stage 5, rearing and falling; Stage 6, full tonic seizures (Figueiredo et al., 2011; Racine, 1972). The rats were observed continuously over 5 hours and were then assessed for seizure activity again at 24 hours.

\subsection{AChE Assays}

AChE assays were done using a modification (Shih et al., 2010) of the Ellman method (Ellman et al., 1961). Briefly, 5\% tissue homogenates from 7 different rat brain regions were prepared in $50 \mathrm{mM}$ phosphate buffer ( $\mathrm{pH}$ 8.00) with $1 \%$ Triton X-100 and centrifuged at 5000 rpm for $10 \mathrm{~min}$. All samples were kept on wet ice and all measurements carried out in triplicate. An aliquot of $10 \mu \mathrm{l}$ of supernatant was added to $100 \mu \mathrm{l}$ of phosphate buffer and $20 \mu \mathrm{l}$ of 5, 5'dithiobis-2-nitrobenzoic acid (DTNB), and endogenous background activity was determined with absorbance at $412 \mathrm{~nm}$ for 10 minutes (30 second intervals) at $37{ }^{\circ} \mathrm{C}$. Acetylthiocholine iodide was then added as substrate and incubation was continued for $40 \mathrm{~min}$ with continuous monitoring of the change in absorption. The final concentrations of both DTNB and acetylthiocholine iodide were $1 \mathrm{mM}$. The change in absorption was found to be linear, and the change in absorption during the 10-20 min period was used to calculate the rate of the reaction after subtracting the corresponding substrate blank values and using the molar extinction coefficient of $13,610 \mathrm{M}^{-1} \mathrm{~cm}^{-1}$.

\subsection{Neuropathology}


Fixation and tissue processing were done 24 hours after paraoxon treatment. Rats were deeply anesthetized with a pentobarbital based preparation $(100 \mathrm{mg} / \mathrm{kg}$, i.p. $)$ and transcardially perfused with PBS $(100 \mathrm{ml})$ followed by $4 \%$ paraformaldehyde $(200 \mathrm{ml})$. Brains were removed and postfixed in $4 \%$ paraformaldehyde overnight at $4{ }^{\circ} \mathrm{C}$, then transferred to a solution of $20 \%$ sucrose in PBS. After 18 hours the sucrose solution was replaced with a fresh $20 \%$ sucrose solution and kept for $72 \mathrm{~h}$, and then the tissues were frozen with dry ice before storage at $-80{ }^{\circ} \mathrm{C}$ until sectioning. Sections containing the hippocampus or amygdala were cut at $40 \mu \mathrm{m}$ on a sliding microtome (Leica Microsystems SM2000R). One series of sections taken every 200 micrometers was mounted on treated slides (Superfrost Plus, Daigger) for silver staining and one adjacent series of coronal sections was mounted on slides for FJB staining. FJB staining (HistoChem, Jefferson, AR) was used to identify irreversibly degenerating neurons in rats with and without oxime treatment. Two-dimensional sample quantitative assessments were made from 6 coronal brain sections $(40 \mu \mathrm{m}$, both hemispheres) per animal from bregma $-3.00 \mathrm{~mm}$ to -4.08 $\mathrm{mm}$. Mounted sections were stained with a $0.0001 \%$ solution of FJB dissolved in $0.1 \%$ acetic acid for 1 hour at $4^{\circ} \mathrm{C}$. Sections were washed in distilled water, dehydrated in ethanol, cleared in xylene, and mounted with DPX. Another set of $40 \mu \mathrm{m}$ sections were collected in $4 \%$ paraformaldehyde and stored at $4{ }^{\circ} \mathrm{C}$ for 5 days. Sections were processed for the detection of degenerated neurons with a silver staining kit (FD Neurosilver kit II; FD NeuroTechnologies, Inc.) according to the manufacturer's instructions. FJB sections were imaged on a Leica AF 6000 system (DMI 6000B) with a 10x objective using Leica LAS-X software. Silver stained sections were imaged on an Olympus BX51 microscope with Olympus DP71 camera and 40x oil objective. Silver stained images were generated using extended depth of field by combining 5 to 8 images taken at different depths within the tissue slices using the maximum local contrast method (Image Pro Plus, version 7.1, Media Cybernetics).

\subsection{Statistics}

Data are reported as means $\pm \mathrm{SD}$. For AChE activity assays, saline and OBD groups were compared using Student's $t$-test for independent samples. Statistical significance was determined using the Holm-Sidak method, with alpha $=5 \%$. Differences were considered significant at $\mathrm{p}<$ 0.05. Racine scale data were compared between OBD and saline at each time point using the nonparametric Mann-Whitney $\mathrm{U}$ test and $p$ values were adjusted using the Holm-Sidak method.

\section{Results}

\subsection{Intranasal bioavailability studies}

In order to investigate the efficacy of intranasal oxime application in bypassing the BBB we used concentrated aqueous solutions of radiolabeled 2-PAM $\left(\left[{ }^{3} \mathrm{H}\right]-2-\mathrm{PAM}, \sim 1.0 \mathrm{~g} / \mathrm{ml}\right)$. 2PAM was chosen for these studies because it is the current standard of treatment for organophosphate poisoning in the US. The $\left[{ }^{3} \mathrm{H}\right]-2-\mathrm{PAM}$ was administered bilaterally to the olfactory sinuses and epithelium of adult rats under anesthesia using an intranasal drug delivery device. We observed detectable levels of $\left[{ }^{3} \mathrm{H}\right]-2-\mathrm{PAM}$ in all brain regions within 2.5 minutes of intranasal application, with increasing concentrations over time (Fig. 2A). Increasing the dose of 2-PAM delivered intranasally led to dose-dependent increases in 2-PAM in all brain regions, with the highest concentrations found in the olfactory bulbs and trigeminal ganglia (Figs. 2B and 2C). We found that giving 3 doses bilaterally at 3 min intervals substantially increased the uptake of 2-PAM into brain parenchyma (data not shown). Decreasing the specific activity of $\left[{ }^{3} \mathrm{H}\right]-2$ - 
PAM without changing the final concentration did not affect uptake of radioactivity into the brain in a proportionate manner indicating a bulk flow mechanism, rather than a saturable uptake system, for intranasal delivery of oximes. However, administration of the highest doses of 2PAM tested (30 mg per animal) resulted in irregular breathing in all rats and respiratory arrest in 5 out of 8 rats given that dose, indicating that high doses of pralidoxime were unsafe for intranasal application. Therefore we tested bioavailability of another potent oxime, OBD, to confirm that it reached all areas of the brain. Using HPLC quantitation we measured OBD concentrations in various brain areas $60 \mathrm{~min}$ after intranasal administration. As expected, the highest concentration of OBD was detected in olfactory bulbs, with varying concentrations in other brain areas similar to the case of $\left[{ }^{3} \mathrm{H}\right]-2-\mathrm{PAM}$ (Fig. 2D).

\subsection{AChE reactivation in brain homogenates}

Organophosphates inactivate AChE through phosphorylation of the enzyme's active site. Oximes reverse organophosphate-mediated AChE inhibition by covalently removing the phosphate moiety of the CTA from the enzyme active site. A limited window of opportunity is available to employ oximes before the AChE phosphorylation, and therefore inhibition, becomes irreversible. We used the paraoxon model of organophosphate poisoning in the rat to assess the feasibility of using intranasal application of oximes as a treatment method, and tested its efficacy in both pre and post-paraoxon administration scenarios. Initially we tested the ability of intranasally administered 2-PAM to reverse paraoxon-induced AChE inhibition by assaying AChE activity in brain samples from rats administered paraoxon. The rats were euthanized 60 min after paraoxon treatment and AChE activity was measured in brain homogenates using an enzyme assay. Enzyme inhibition ranged from 80 to $90 \%$ in brain homogenates from different brain regions $60 \mathrm{~min}$ after subcutaneous injection of paraoxon. The intranasal administration of lower levels of 2-PAM (10 mg per animal) that were well tolerated was generally ineffective at reducing AChE inactivation except in the olfactory bulbs, where partial reactivation was observed (data not shown).

Subsequent testing of OBD via intranasal administration under similar conditions showed that it was capable of partially reducing paraoxon-mediated AChE inhibition in all brain areas examined. Pre-treatment with intranasal OBD $30 \mathrm{~min}$ before paraoxon administration resulted in partial reactivation of $\mathrm{AChE}$ activity, but the reactivation only reached significance in the olfactory bulbs (Fig. 3A). However, intranasal treatment with OBD 5 min after paraoxon administration resulted in significant enzyme activity recovery in all areas tested (Fig. 3B). Intranasal OBD treatment brought paraoxon-inhibited enzyme activity back to almost $90 \%$ of control values in the olfactory bulbs, whereas enzyme activity recovery in other brain regions ranged from about 4 to $5 \%$ in the hippocampus and cortex, to $13 \%$ in the cerebellum. No toxic effects were seen with intranasal OBD at high doses (38 $\mathrm{mg}$ per animal). The regional pattern of AChE activity recovery (Fig. 3) corresponded fairly well to the regional bioavailability data for intranasal OBD (Fig. 2D) consistent with a direct transport of OBD from the nasal mucosa to the brain thus bypassing the BBB.

\subsection{Seizure reduction and neuropathology}

Based on the AChE enzyme assay results indicating that some recovery of enzyme function was present, we investigated intranasal application of OBD in the paraoxon model to determine if this method was capable of reducing seizure severity and preventing neurodegeneration. We used high doses of paraoxon in this study $(4 \mathrm{mg} / \mathrm{kg})$ to model severe 
organophosphate poisoning (Deshpande et al., 2014). Paraoxon administered at this dose does not directly cause neuronal death in the CNS, but instead results in prolonged SE, which leads to widespread excitotoxic neuronal death (Deshpande et al., 2014; Todorovic et al., 2012). For this set of experiments the same rats were used for both seizure observations and neuropathology. Rats were assigned randomly to 4 groups (saline or OBD 30 minutes pre-treatment and saline or OBD 5 minutes post-treatment relative to paraoxon administration) with 10 rats per group (total $=40)$.

\subsubsection{OBD pre-treatment 30 minutes prior to paraoxon administration}

Using light isoflurane anesthesia, animals were administered OBD or saline intranasally, 30 minutes before paraoxon administration. Paraoxon administration was immediately followed (1 minute or less) by the standard treatment of intramuscular injections of $2-\mathrm{PAM}$ at $25 \mathrm{mg} / \mathrm{kg}$ and atropine sulphate at $2 \mathrm{mg} / \mathrm{kg}$. Under these conditions subcutaneous paraoxon administration at $4 \mathrm{mg} / \mathrm{kg}$ in rats induced observable seizure activity within 2 to 4 minutes. By 10 minutes postparaoxon administration rats in both the saline treated and OBD treated groups had reached stage 3 on the modified Racine scale of seizure severity (Figueiredo et al., 2011; Racine, 1972) (Fig. 4A). Seizure severity remained high over the course of 5-7 hours (stage 3 or greater) in the saline treated group, whereas the OBD treated group showed progressively diminished seizure activity during this time period.

At 24 hours after paraoxon administration $36 \%$ of the saline treated rats had died, and all remaining rats in this group exhibited continued seizures at stages 1 to 2 on the modified Racine scale. In contrast, all the OBD treated rats survived and appeared normal with no observable seizure activity at 24 hours (see Table 1 below). The surviving rats were then sacrificed and neurodegeneration was determined using Fluoro-Jade B (FJB) staining. We chose the 24 hour time point because we have previously found FJB staining to be maximal at this time after organophosphate poisoning (Prager et al., 2014). The prolonged seizures in the saline treated group resulted in extensive excitotoxic neuronal death in many susceptible regions including midline thalamic nuclei, basomedial and basolateral amygdaloid nuclei, and perirhinal cortex. Other areas such as piriform cortex, hippocampus, dorsolateral thalamus and layers II-IV in parietal cortex had variable staining between saline-treated animals, with some rats exhibiting widespread neuronal degeneration throughout these areas (Fig. 5). In contrast, no detectable degenerating neurons were observed at 24 hours in any area in any of the animals treated intranasally with OBD 30 min prior to paraoxon administration (Fig. 5).

\subsubsection{OBD post-treatment 5 minutes after paraoxon administration}

Having determined that treatment with OBD prior to paraoxon administration was effective at reducing seizures and preventing neuropathology, we subsequently studied the effect of intranasal OBD treatment 5 min after paraoxon administration. In this group of animals neurodegeneration was evaluated by using both FJB and silver staining 24 hours after paraoxon administration (Figs. 5-7), and seizure severity was followed for 24 hours using the modified Racine scale (Fig. 4B). The mortality rate for the saline-treated group at 24 hours in this set of experiments was $47 \%$, whereas no rats in the OBD-treated group died (Table 1). As in the case of pre-treatment with OBD, post-treatment after paraoxon administration increased AChE enzyme activity and prevented SE, mortality and neuronal degeneration.

\subsection{Total mortality rates for the entire study.}


Throughout the course of the study a total of 104 rats were given paraoxon and treated intranasally with either saline or $38 \mathrm{mg}$ of OBD and observed for 24 hours (saline $=58, \mathrm{OBD}=$ 46, Table 1). Mortality rates across saline treated groups averaged $41 \%$ at 24 hours, whereas no rats in the two OBD treated groups had died by 24 hours, the time at which the rats were sacrificed for neuropathology assessments.

\section{Discussion}

Our results demonstrate that intranasal delivery of oximes is a viable method for preventing SE caused by severe organophosphate poisoning, as well as the resultant neurodegenerative sequelae. "Nose-to-brain" delivery has gained increasing attention as a method for bypassing the BBB to rapidly deliver drugs to the CNS along the olfactory and trigeminal neural pathways (Lochhead and Thorne, 2012). Our results further confirm that small molecules such as OBD gain sufficient access to brain parenchyma after intranasal application that they can counteract some of the AChE inhibition at cholinergic synapses caused by organophosphate poisoning. Partial recovery of enzyme activity was observed in all regions of the CNS tested, including cervical spinal cord, and this level of enzyme recovery was sufficient to reduce seizure activity, increase animal survival to $100 \%$, and completely prevent neuronal loss. The prevention of mortality and neuronal sparing were similar under OBD pre-treatment and post-treatment scenarios indicating relatively wide latitude in the window of effectiveness. Future studies will need to determine the full window of opportunity for intranasal oxime treatment.

We found intranasal OBD administration to be much more effective at reducing paraoxon-mediated AChE inhibition in the rat brain than 2-PAM. The finding that intranasal 2PAM administration had a relatively low efficiency in reducing paraoxon-mediated AChE inactivation in rat brain homogenates is consistent with the literature showing that OBD is much more effective at reactivating rat brain AChE than 2-PAM (Atanasov et al., 2013; Kuca et al., 2009). However it is difficult to reconcile the modest AChE reactivation we observed with intranasal OBD administration (Fig 3) with the large effect on reducing seizures (Fig. 4), and protection against mortality (Table 1) and neuronal cell loss (Figs. 5-7). It is possible that in vitro measurement of $\mathrm{AChE}$ activity in brain homogenates underestimated the reactivation in vivo because remaining paraoxon in the homogenates continued to inhibit enzyme activity. It is also possible that partial reactivation of AChE activity in specific brain regions that have a role in generation and spreading of seizure activity can prevent SE as suggested by studies with the organophosphate nerve agent soman (Prager et al., 2013). These studies showed that AChE activity levels in seizurogenic brain regions including the basolateral amygdala were critical for induction of seizure activity in response to soman. It is possible that modest AChE reactivation in AChE-rich regions such as the basolateral amygdala in response to intranasal OBD administration could prevent prolonged seizures and protect the CNS from excitotoxic injury after organophosphate poisoning.

The brain has a unique lymphatic system (Louveau et al., 2015), wherein paravascular spaces surrounding elements of the cerebral vasculature act as specialized conduits allowing for long distance movement of water and dissolved solutes. This paravascular system, which has been termed the "glymphatic system" because of the involvement of astrocytes (Iliff et al., 2012; Thrane et al., 2014), allows for the transport of fluids and dissolved solutes from the olfactory mucosa to distant regions of the CNS. The glymphatic system associated with the olfactory and trigeminal pathways has been exploited for the delivery of numerous drugs and hormones to the 
CNS in various therapeutic applications (Casettari and Illum, 2014; Dhuria et al., 2010; Djupesland et al., 2014; Hanson and Frey, 2008; Liu et al., 2001; Lochhead et al., 2015). We took advantage of the glymphatic system's ability to transport solutes in bulk to rapidly deliver oximes to the brain to counteract organophosphate poisoning. A rapid and non-invasive method of protecting humans from organophosphates and of treating humans who have just been exposed to them would be advantageous in cases of organophosphate insecticide poisoning, or CTA release events involving civilians and military personnel worldwide. Intranasally administered OBD reaches the cortex and other brain regions within minutes and is effective at reducing the acute and chronic adverse CNS effects of paraoxon-induced AChE inhibition. Current attempts to overcome the BBB have focused on techniques such as chemical modification of oximes to reduce their hydrophilicity and increase their capacity to penetrate the barrier (Kovarik et al., 2013). We have shown that intranasal administration is an alternative method of bypassing the BBB. Intranasal oxime administration provides advantages relative to existing therapies including rapid, non-invasive delivery to the brain, which we have shown is moderately effective at reducing central AChE inactivation and very effective at reducing seizures and protecting neurons from damage caused by excess cholinergic activation. We have also shown that repeated applications are well tolerated and act to increase delivery of oxime to brain parenchyma.

Insecticide poisoning deaths worldwide have been estimated to number in the hundreds of thousands per year, mostly due to intentional ingestion of pesticides in attempted suicides (Gunnell et al., 2007). Many of these cases involve ingestion of large quantities of insecticides, including organophosphate-based insecticides, which can remain in the system for days, potentially causing long term CNS damage (Hrabetz et al., 2013). In severe cases like this, as an adjunct to the standard therapy of atropine plus oxime, intranasal obidoxime treatment could be given at regular intervals until blood cholinesterase levels return to normal, providing protection for the CNS. Intranasal obidoxime could be used in place of, or in conjunction with second generation benzodiazepines such as midazolam to prevent seizures and subsequent brain damage (Shrot et al., 2014). Nasal inhalers with specific oximes or combinations of oximes may be practical additions to combat personnel emergency kits that include atropine/oxime autoinjectors for treating organophosphate poisoning. The simplicity of nasal application and the rapid delivery of oximes to the brain make this method especially useful for self-administration where access to a hospital may be delayed. Because we found that pre-treatment 30 minutes before, or post-treatment 5 minutes after exposure to paraoxon were equally effective, nasal administration could be used in both prophylactic and post-exposure situations making it a versatile tool in treating organophosphate poisoning.

\section{Acknowledgements}

We acknowledge Impel NeuroPharma, Inc. Seattle, WA for making their POD intranasal delivery device available for our use. The authors have no competing interests to declare. The opinions or assertions contained herein are the private views of the authors, and are not to be construed as reflecting the views of the Department of Defense. This work was supported by the NIH grant NS076448. Graphical abstract adapted from (Swanson, 2004) (http://larrywswanson.com/?page id=164).

\section{References}


Atanasov, V. N., Petrova, I., and Dishovsky, C. (2013). In vitro investigation of efficacy of new reactivators on OPC inhibited rat brain acetylcholinesterase. Chem. Biol. Interact. 203, 139-143.

Balan, A., Barness, I., and Amitai, G. (1993). Synthesis of tritium labelled oximes: 2-pyridine aldoxime methiodide (2-PAM) and 1-(2-hydroxyiminomethylpyridinium)-1-(4carboxyamidopyridinium)dimethylether dichloride (HI-6), with high specific activity. J. Label. Compd. Radiopharm 33, 19-32.

Buckley, N. A., Eddleston, M., Li, Y., Bevan, M., and Robertson, J. (2011). Oximes for acute organophosphate pesticide poisoning. Cochrane. Database. Syst. Rev. CD005085.

Casettari, L. and Illum, L. (2014). Chitosan in nasal delivery systems for therapeutic drugs. J. Control. Release. 190, 189-200.

Deshpande, L. S., Carter, D. S., Phillips, K. F., Blair, R. E., and Delorenzo, R. J. (2014). Development of status epilepticus, sustained calcium elevations and neuronal injury in a rat survival model of lethal paraoxon intoxication. Neurotoxicology. 44, 17-26.

Dhuria, S. V., Hanson, L. R., and Frey, W. H. (2010). Intranasal delivery to the central nervous system: mechanisms and experimental considerations. J. Pharm. Sci. 99, 1654-1673.

Djupesland, P. G., Messina, J. C., and Mahmoud, R. A. (2014). The nasal approach to delivering treatment for brain diseases: an anatomic, physiologic, and delivery technology overview. Ther. Deliv. 5, 709-733.

Eddleston, M., Eyer, P., Worek, F., Juszczak, E., Alder, N., Mohamed, F., Senarathna, L., Hittarage, A., Azher, S., Jeganathan, K., Jayamanne, S., Von, M. L., Dawson, A. H., Sheriff, M. H., and Buckley, N. A. (2009). Pralidoxime in acute organophosphorus insecticide poisoning--a randomised controlled trial. PLoS. Med. 6, e1000104.

Ellman, G. L., Courtney, K. D., Andres, V., Jr., and Feather-Stone, R. M. (1961). A new and rapid colorimetric determination of acetylcholinesterase activity. Biochem. Pharmacol. 7, 88-95.

Eyer, F., Worek, F., Eyer, P., Felgenhauer, N., Haberkorn, M., Zilker, T., and Thiermann, H. (2009). Obidoxime in acute organophosphate poisoning: 1 - clinical effectiveness. Clin. Toxicol. (Phila) 47, 798-806.

Figueiredo, T. H., Aroniadou-Anderjaska, V., Qashu, F., Apland, J. P., Pidoplichko, V., Stevens, D., Ferrara, T. M., and Braga, M. F. (2011). Neuroprotective efficacy of caramiphen against soman and mechanisms of its action. Br. J. Pharmacol. 164, 1495-1505.

Gunnell, D., Eddleston, M., Phillips, M. R., and Konradsen, F. (2007). The global distribution of fatal pesticide self-poisoning: systematic review. BMC. Public. Health. 7, 357.

Hanson, L. R. and Frey, W. H. (2008). Intranasal delivery bypasses the blood-brain barrier to target therapeutic agents to the central nervous system and treat neurodegenerative disease. BMC. Neurosci. 9 Suppl 3, S5. 
Hrabetz, H., Thiermann, H., Felgenhauer, N., Zilker, T., Haller, B., Nahrig, J., Saugel, B., and Eyer, F. (2013). Organophosphate poisoning in the developed world - a single centre experience from here to the millennium. Chem. Biol. Interact. 206, 561-568.

Iliff, J. J., Wang, M., Liao, Y., Plogg, B. A., Peng, W., Gundersen, G. A., Benveniste, H., Vates, G. E., Deane, R., Goldman, S. A., Nagelhus, E. A., and Nedergaard, M. (2012). A paravascular pathway facilitates CSF flow through the brain parenchyma and the clearance of interstitial solutes, including amyloid beta. Sci. Transl. Med 4, 147ra111.

Jett, D. A. (2007). Neurological aspects of chemical terrorism. Ann. Neurol. 61, 9-13.

Kovarik, Z., Macek, N., Sit, R. K., Radic, Z., Fokin, V. V., Barry, S. K., and Taylor, P. (2013). Centrally acting oximes in reactivation of tabun-phosphoramidated AChE. Chem. Biol. Interact. 203, 77-80.

Kuca, K., Musilova, L., Palecek, J., Cirkva, V., Paar, M., Musilek, K., Hrabinova, M., Pohanka, M., Karasova, J. Z., and Jun, D. (2009). Novel bisquaternary oximes--reactivation of acetylcholinesterase and butyrylcholinesterase inhibited by paraoxon. Molecules. 14, 4915-4921.

Liu, X. F., Fawcett, J. R., Thorne, R. G., and Frey, W. H. (2001). Non-invasive intranasal insulin-like growth factor-I reduces infarct volume and improves neurologic function in rats following middle cerebral artery occlusion. Neurosci. Lett. 308, 91-94.

Lochhead, J. J. and Thorne, R. G. (2012). Intranasal delivery of biologics to the central nervous system. Adv. Drug Deliv. Rev. 64, 614-628.

Lochhead, J. J., Wolak, D. J., Pizzo, M. E., and Thorne, R. G. (2015). Rapid transport within cerebral perivascular spaces underlies widespread tracer distribution in the brain after intranasal administration. J. Cereb. Blood. Flow. Metab. 35, 371-381.

Louveau, A., Smirnov, I., Keyes, T. J., Eccles, J. D., Rouhani, S. J., Peske, J. D., Derecki, N. C., Castle, D., Mandell, J. W., Lee, K. S., Harris, T. H., and Kipnis, J. (2015). Structural and functional features of central nervous system lymphatic vessels. Nature. 523, 337-341.

McDonough, J. H., Jr. and Shih, T. M. (1997). Neuropharmacological mechanisms of nerve agent-induced seizure and neuropathology. Neurosci. Biobehav. Rev. 21, 559-579.

Peter, J. V., Moran, J. L., and Graham, P. (2006). Oxime therapy and outcomes in human organophosphate poisoning: an evaluation using meta-analytic techniques. Crit. Care Med. 34, 502-510.

Prager, E. M., Aroniadou-Anderjaska, V., Almeida-Suhett, C. P., Figueiredo, T. H., Apland, J. P., and Braga, M. F. (2013). Acetylcholinesterase inhibition in the basolateral amygdala plays a key role in the induction of status epilepticus after soman exposure. Neurotoxicology. 38, 84-90.

Prager, E. M., Aroniadou-Anderjaska, V., Almeida-Suhett, C. P., Figueiredo, T. H., Apland, J. P., Rossetti, F., Olsen, C. H., and Braga, M. F. (2014). The recovery of acetylcholinesterase activity and the progression of neuropathological and pathophysiological alterations in the rat 
basolateral amygdala after soman-induced status epilepticus: relation to anxiety-like behavior. Neuropharmacology 81, 64-74.

Racine, R. J. (1972). Modification of seizure activity by electrical stimulation. II. Motor seizure. Electroencephalogr. Clin. Neurophysiol. 32, 281-294.

Shih, T. M., Skovira, J. W., O'Donnell, J. C., and McDonough, J. H. (2010). In vivo reactivation by oximes of inhibited blood, brain and peripheral tissue cholinesterase activity following exposure to nerve agents in guinea pigs. Chem. Biol. Interact. 187, 207-214.

Shrot, S., Ramaty, E., Biala, Y., Bar-Klein, G., Daninos, M., Kamintsky, L., Makarovsky, I., Statlender, L., Rosman, Y., Krivoy, A., Lavon, O., Kassirer, M., Friedman, A., and Yaari, Y. (2014). Prevention of organophosphate-induced chronic epilepsy by early benzodiazepine treatment. Toxicology. 323, 19-25.

SWANSON,L.W. (2004). Brain maps: structure of the rat brain: Third Edition. Elsevier: Amsterdam.

Thiermann, H., Mast, U., Klimmek, R., Eyer, P., Hibler, A., Pfab, R., Felgenhauer, N., and Zilker, T. (1997). Cholinesterase status, pharmacokinetics and laboratory findings during obidoxime therapy in organophosphate poisoned patients. Hum. Exp. Toxicol. 16, 473-480.

Thorne, R. G., Pronk, G. J., Padmanabhan, V., and Frey, W. H. (2004). Delivery of insulin-like growth factor-I to the rat brain and spinal cord along olfactory and trigeminal pathways following intranasal administration. Neuroscience. 127, 481-496.

Thrane, A. S., Rangroo, T., V, and Nedergaard, M. (2014). Drowning stars: reassessing the role of astrocytes in brain edema. Trends. Neurosci. 37, 620-628.

Todorovic, M. S., Cowan, M. L., Balint, C. A., Sun, C., and Kapur, J. (2012). Characterization of status epilepticus induced by two organophosphates in rats. Epilepsy Res. 101, 268-276.

Wille, T., Kaltenbach, L., Thiermann, H., and Worek, F. (2013). Investigation of kinetic interactions between approved oximes and human acetylcholinesterase inhibited by pesticide carbamates. Chem. Biol. Interact. 206, 569-572.

Zdarova, K. J., Novotny, L., Antos, K., Zivna, H., and Kuca, K. (2010). Time-dependent changes in concentration of two clinically used acetylcholinesterase reactivators (HI-6 and obidoxime) in rat plasma determined by HPLC techniques after in vivo administration. Anal. Sci. 26, 63-67. 
Table 1. Mortality rates in treatment groups

\begin{tabular}{l|lll}
\hline Intranasal Treatment & \# animals & deaths & $\%$ mortality \\
\hline OBD pretreatment & 22 & 0 & $0 \%$ \\
OBD post-treatment & 24 & 0 & $0 \%$ \\
Total OBD & 46 & 0 & $0 \%$ \\
Saline pretreatment & 28 & 10 & $36 \%$ \\
Saline post-treatment & 30 & 14 & $47 \%$ \\
Total saline & 58 & 24 & $41 \%$ \\
\hline
\end{tabular}

Table 1. Mortality rates within $\sim 24 \mathrm{hr}$ for rats given $4 \mathrm{mg} / \mathrm{kg}$ paraoxon (AChE enzyme reactivation and neuropathology studies). All rats received atropine sulphate and 2-PAM via intramuscular injection immediately after paraoxon administration. The only difference between groups was the intranasal treatment with saline or OBD. Total rats in the study $=104$.

\section{Figure Legends:}

Fig. 1. Schematic of experimental protocol. The experimental design for the efficacy studies with OBD is given in a simplified schematic. Two parallel sets of experiments were done using the same basic protocol. In both sets of experiments, rats were treated intranasally with either OBD (38 mg) or saline, administered bilaterally. Treatment groups were administered OBD or saline intranasally either $30 \mathrm{~min}$ prior to (A), or 5 min after paraoxon administration (B). After paraoxon administration (time 0), all animals were treated immediately with intramuscular 2PAM and atropine sulphate. In one set of experiments (blue timeline) animals were observed behaviorally for 5 hours after paraoxon administration, and assessed again at 24 hours, at which time they were sacrificed for neuropathology assessments. In another set of experiments (red timeline), rats were not observed behaviorally, and were sacrificed 1 hour after paraoxon administration for $\mathrm{AChE}$ determinations in various brain regions.

Fig. 2. Bioavailability studies. Time course of radiolabeled 2-PAM uptake in the brain (A). The distribution of $\left[{ }^{3} \mathrm{H}\right]-2-\mathrm{PAM}$ in blood and various brain regions using a single dose of $1.0 \mathrm{mg}$. (note: animals were not perfused prior to dissecting tissues in the experiment shown in panel A in order to collect blood for analysis; $n=2$ for 2.5 and 5 min groups, $n=3$ for 30 and 60 min groups). Effect of increasing the dose of 2-PAM per administration on the concentrations achieved in various brain regions $30 \mathrm{~min}$ after intranasal administration ( 3 administrations at 3 minute intervals; applied dose range $=1 \mathrm{mg}$ total to $30 \mathrm{mg}$ total). The highest concentrations were observed in the olfactory bulb and trigeminal ganglia and nerve (B). At the highest dose used $(30 \mathrm{mg})$ the concentration attained in other brain areas ranged between approximately 18 $\mu \mathrm{M}$ in hippocampus to $38 \mu \mathrm{M}$ in the cervical spinal cord (C) (B and $\mathrm{C} ; \mathrm{n}=3$ ). OBD uptake and retention in the brain after intranasal application (D). HPLC quantitation of OBD was done to confirm uptake and retention in brain parenchyma 60 min after intranasal administration $(\mathrm{D} ; \mathrm{n}=$ $6)$.

Fig. 3. Reduction of $\mathrm{AChE}$ inhibition in response to intranasal OBD administration. Intranasal delivery of OBD either $30 \mathrm{~min}$ prior to (A) or 5 min after paraoxon administration (B) partially reduced paraoxon mediated $\mathrm{AChE}$ inhibition in the brain. AChE activity was 
determined at $60 \mathrm{~min}$ after paraoxon administration. AChE activity values in control rats $(\mu \mathrm{mol} / \mathrm{min} / \mathrm{g}$ wet tissue $)$; olfactory bulbs $=8.7 \pm 1.5$, frontal cortex $=16.2 \pm 1.3$, hippocampus $=$ $14.4 \pm 2.2$, cerebral cortex $=26.1 \pm 3.9$, midbrain $=34.3 \pm 1.4$, cerebellum $=8.4 \pm 3.5$, brainstem $=$ $24 \pm 1.8$ (means expressed as $\%$ control $\pm \mathrm{SD}, \mathrm{n}=6$ per group, $* * \mathrm{p}<0.05, * \mathrm{p}<0.01$ relative to saline treatment).

Fig. 4. Seizure reduction in response to intranasal OBD administration. Intranasal delivery of OBD (38 mg) $30 \mathrm{~min}$ prior to (A) or 5 min after (B) paraoxon administration $(4 \mathrm{mg} / \mathrm{kg})$ reduced seizure activity in rats. Seizure activity was monitored independently by two trained researchers. Prolonged seizures induced by AChE inhibition are responsible for the neurodegeneration resulting from organophosphate poisoning (means $\pm \mathrm{SD}, \mathrm{n}=10$ per group). In the pre-administration scenario, all differences between the OBD and saline groups from 15 min onward were significant $(\mathrm{p}<0.01)$. In the post-administration condition all differences between the OBD and saline groups were significant from 10 min onward $(\mathrm{p}<0.01)$.

Fig. 5. Neuropathology in saline and OBD treated animals using FJB staining. Pre-treatment: Intranasal delivery of $38 \mathrm{mg}$ OBD 30 minutes prior to paraoxon treatment completely prevented the neurodegeneration observed using FJB staining at 24 hours in the saline treated rats. Neurodegeneration in the hippocampus ( $\mathrm{A}$ and $\mathrm{E}$ ) and neocortex ( $\mathrm{C}$ and $\mathrm{G}$ ) was not seen consistently in all saline-treated rats given paraoxon. Neurodegeneration in these areas was not observed in any of the OBD treated rats given paraoxon $(\mathrm{B}, \mathrm{D} F$ and $\mathrm{H})$. Abbreviations: $\mathrm{I}-\mathrm{V}=$ layers $\mathrm{I}$ through $\mathrm{V}$ of neocortex, $\mathrm{CA} 3=\mathrm{CA} 3$ region of hippocampus, $\mathrm{gr}=$ granule cell layer of the dentate gyrus, $\mathrm{pm}=$ the polymorph layer of the dentate gyrus, $\mathrm{PRh}=$ perirhinal cortex. Posttreatment: Intranasal administration of OBD immediately after paraoxon treatment completely prevented the neurodegeneration observed using FJB staining at 24 hours in saline treated rats. The left column (I, K, M and O) shows FJB staining in saline-treated rats, whereas the right column (J, L, N and P) shows the same areas in rats treated intranasally with OBD 5 min after paraoxon administration ( $\mathrm{n}=10$ per group). Abbreviations: $\mathrm{III}=$ third ventricle, $\mathrm{BM}=$ basomedial amygdala, $\mathrm{MD}=$ mediodorsal thalamic nucleus, $\mathrm{PIR}=$ piriform cortex, $\mathrm{PVT}=$ paraventricular thalamic nucleus, $\mathrm{Re}=$ nucleus reuniens.

Fig. 6. Neuropathology in cortex and hippocampus $24 \mathrm{hr}$ after paraoxon administration using silver staining. Degenerating neurons (neurons covered with extensive black silver grains) were observed throughout the brains of saline-treated rats 24 hours after paraoxon administration. No similarly stained neurons were observed in rats treated with OBD 5 min after paraoxon administration. Panels A (intranasal saline) and B (intranasal OBD) show neuronal degeneration in the granule cell layer of the dentate gyrus. Panels $C$ (saline) and D (OBD) depict silver staining in layer VI of temporal cortex (perirhinal cortex), whereas panels E (saline) and F (OBD) show staining in the fascicola cinerea of the hippocampus (40x objective, extended depth of field, $\mathrm{n}=4$ saline, 3 OBD).

Figure 7. Neuropathology in amygdala, cortex and nucleus reuniens $24 \mathrm{hr}$ after paraoxon administration using silver staining. Panels A (saline-treated) and B (OBD treated) show silver staining in the medial amygdala. In some rats treated with OBD intranasally 5 min after paraoxon, faint silver staining was seen on some neurons in susceptible areas such as the medial amygdala (arrow in B) indicating that the neurons underwent stress due to brief excitotoxicity, 
but were not in the process of degenerating. Panels $\mathrm{C}$ (saline) and D (OBD) depict silver staining in layer III of somatosensory cortex. Panels E (saline) and F (OBD) show silver staining in the reuniens nucleus (40x objective, enhanced depth of field). 
Mofdified Racine Scale seizure severity observations

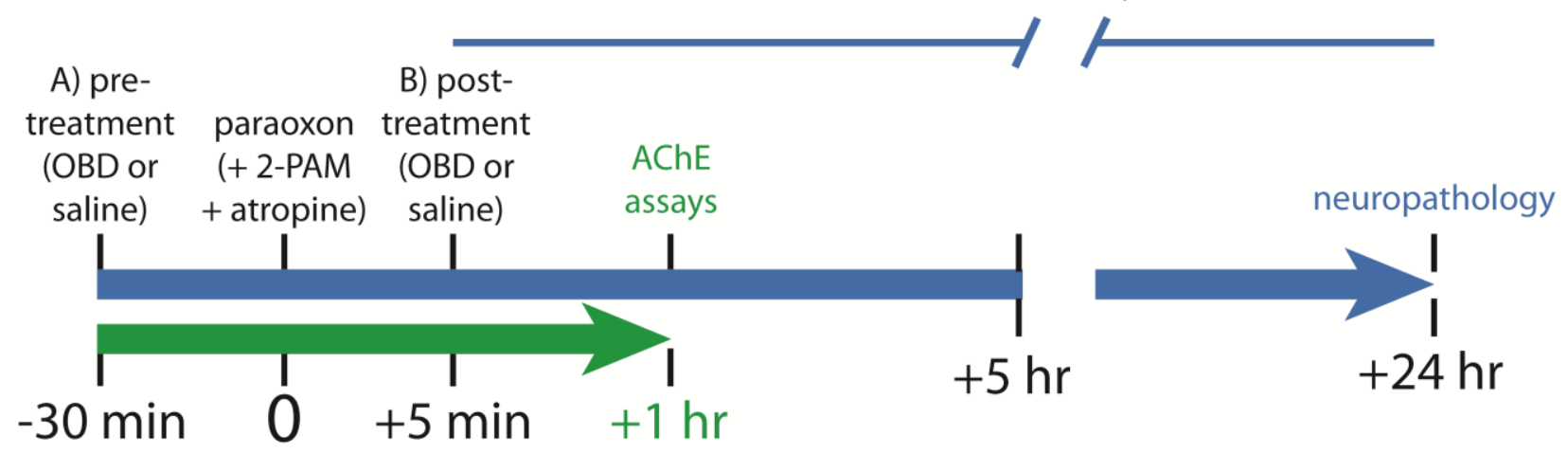



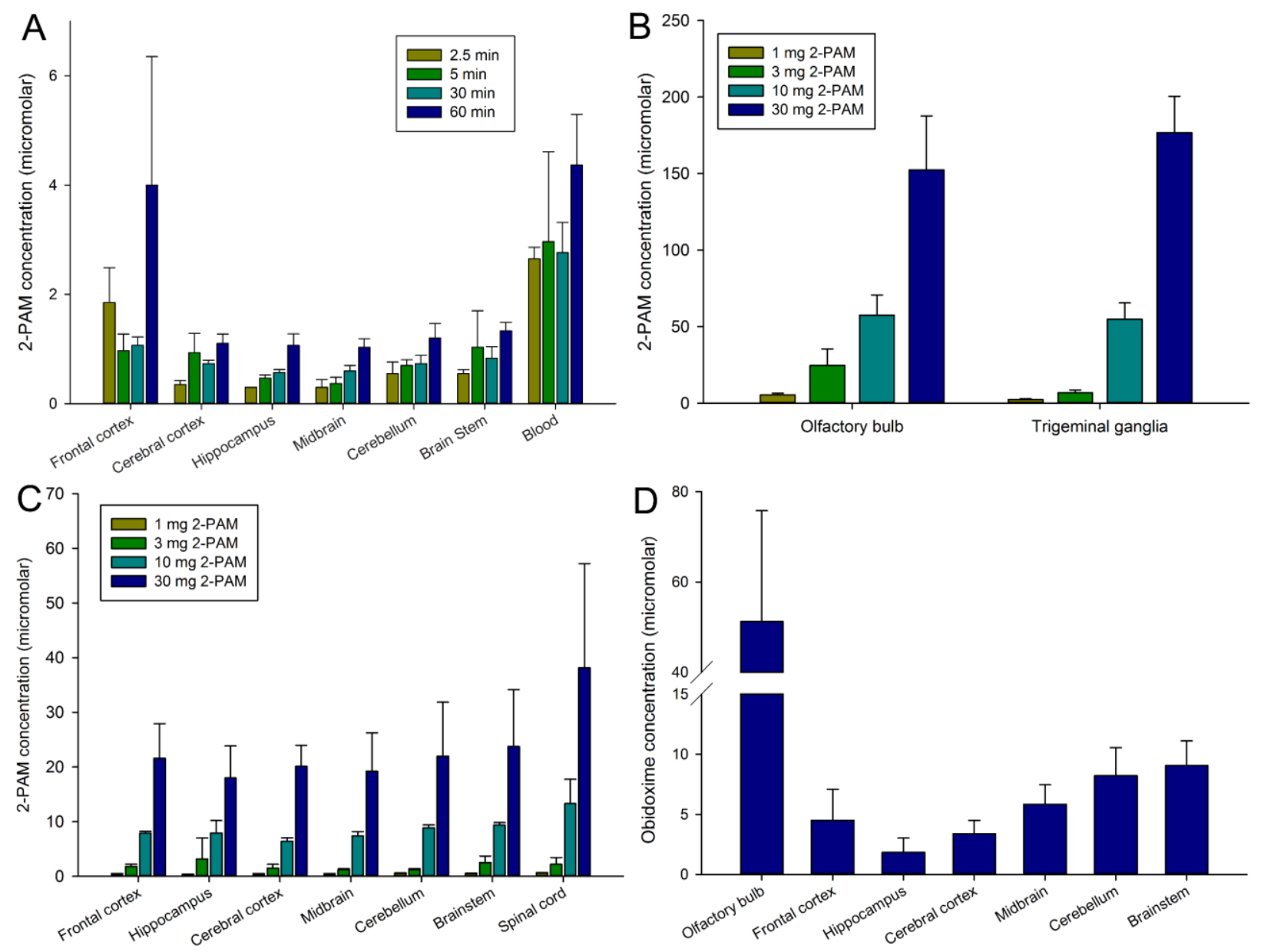


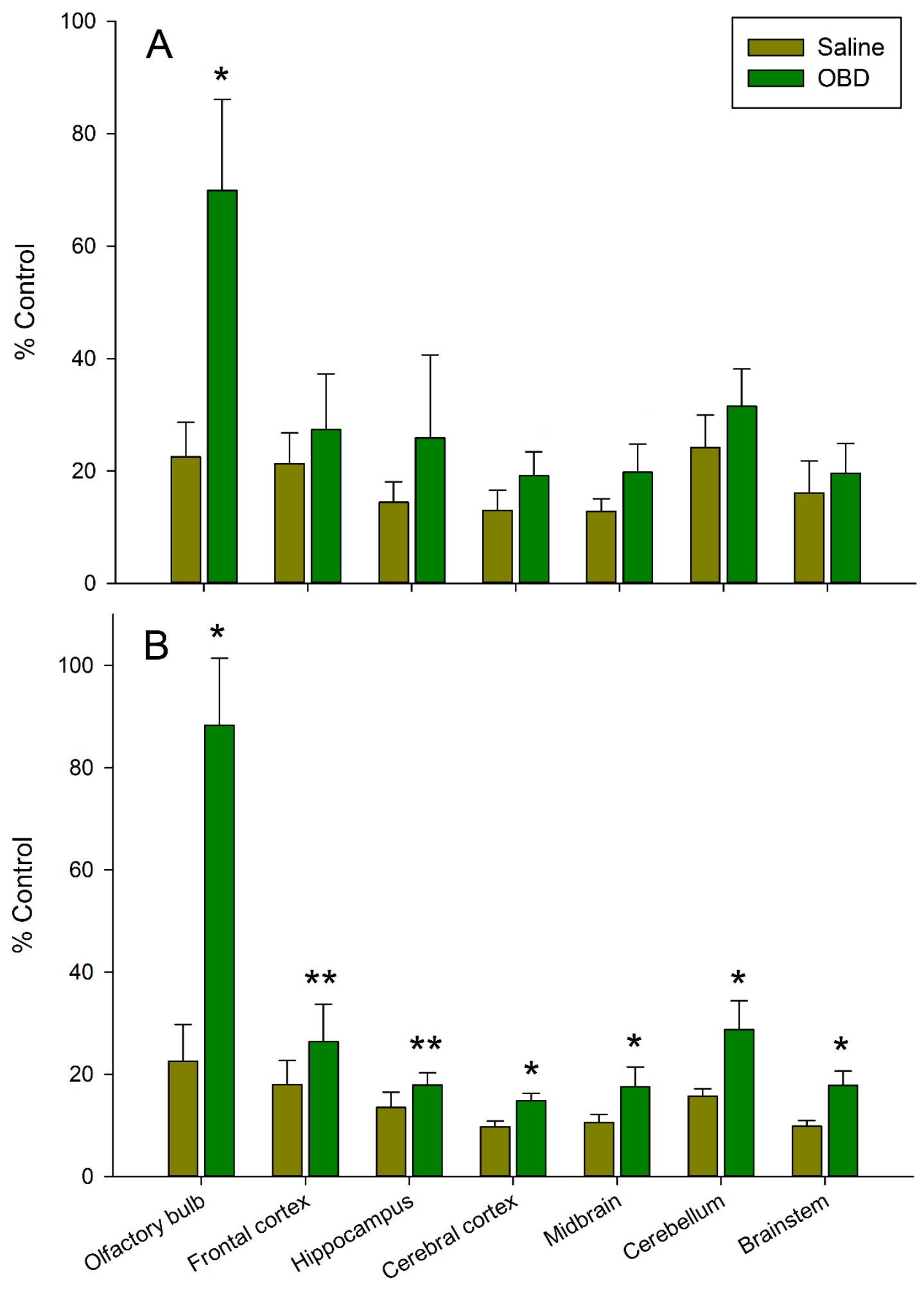



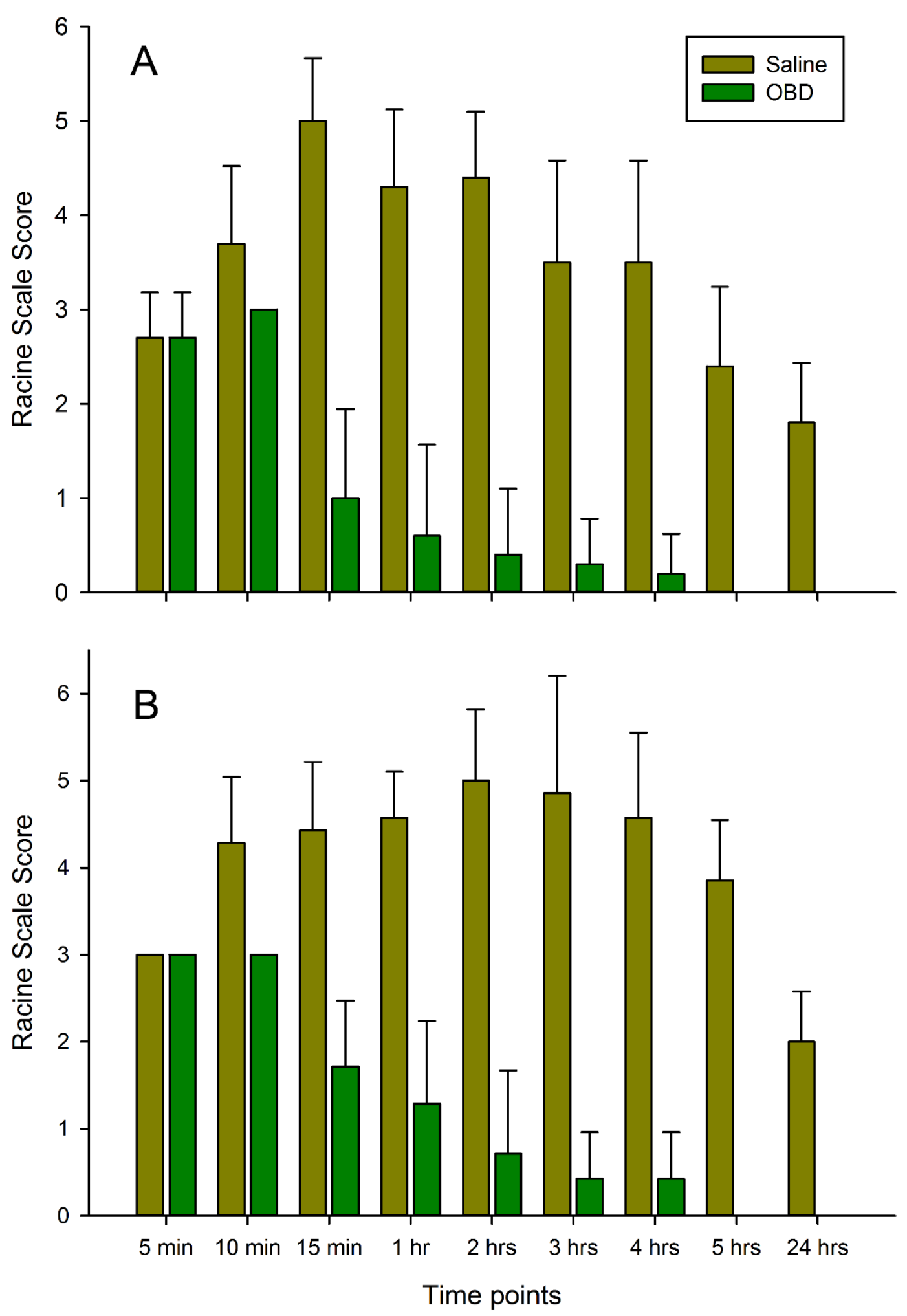


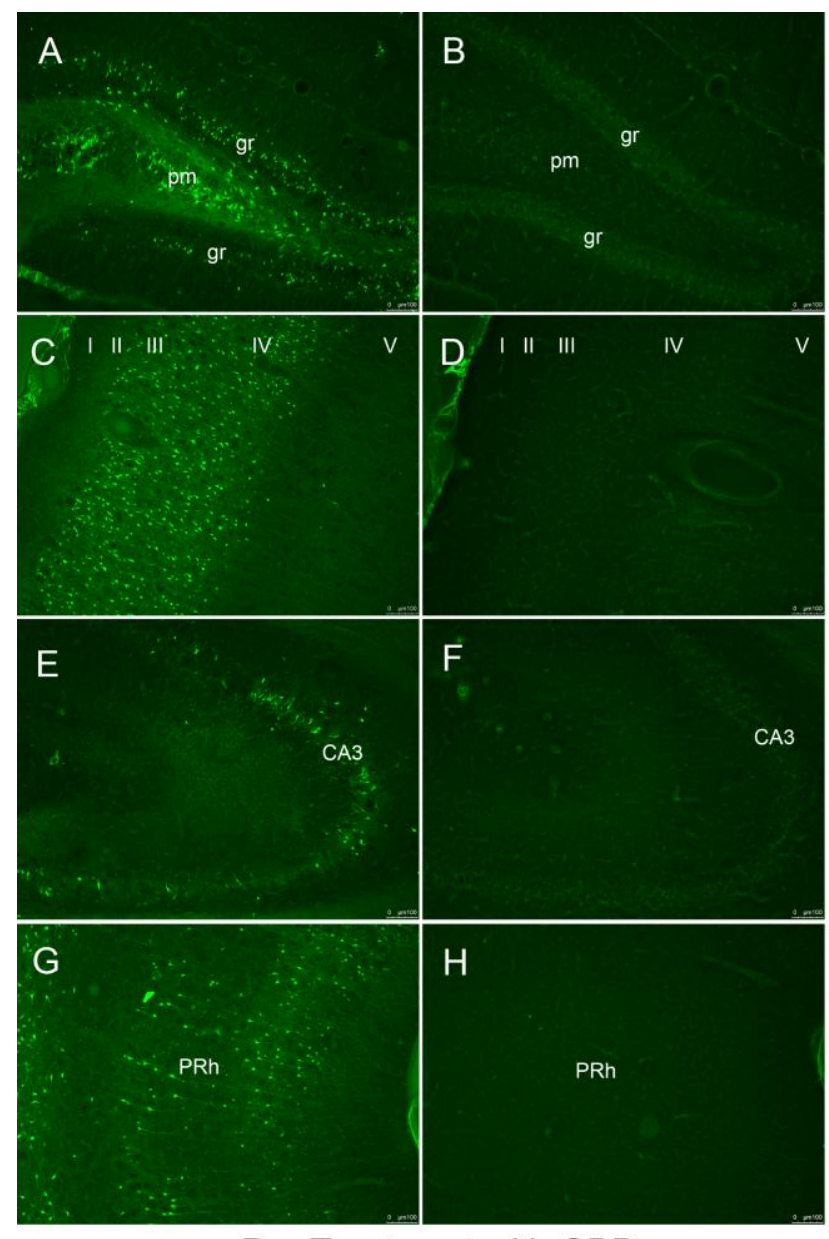

Pre-Treatment with OBD

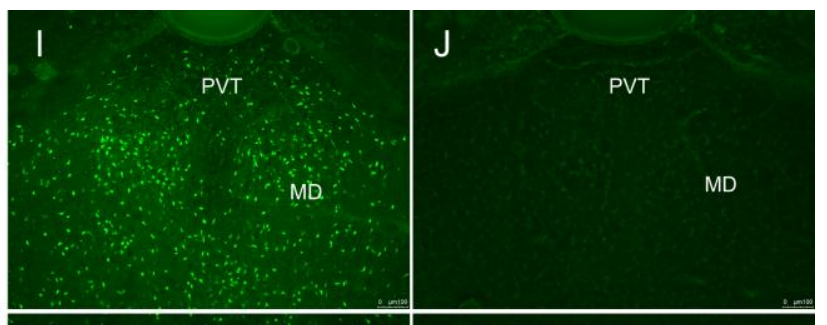

$\mathrm{Re}$

$\operatorname{Re}$

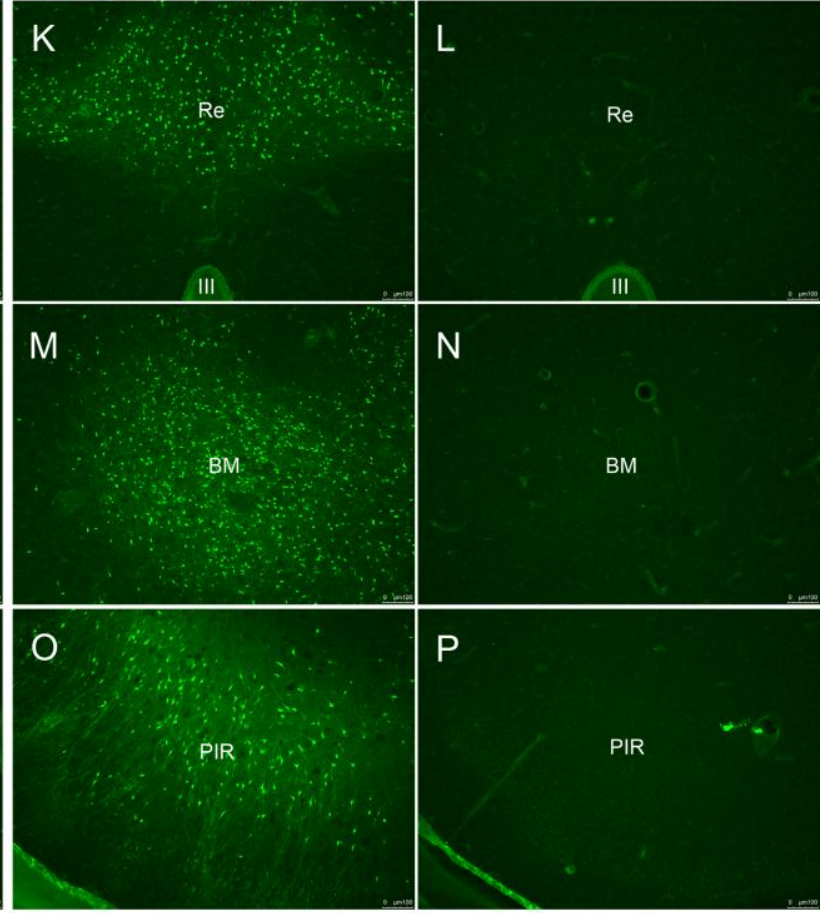

Post-treatment with OBD 


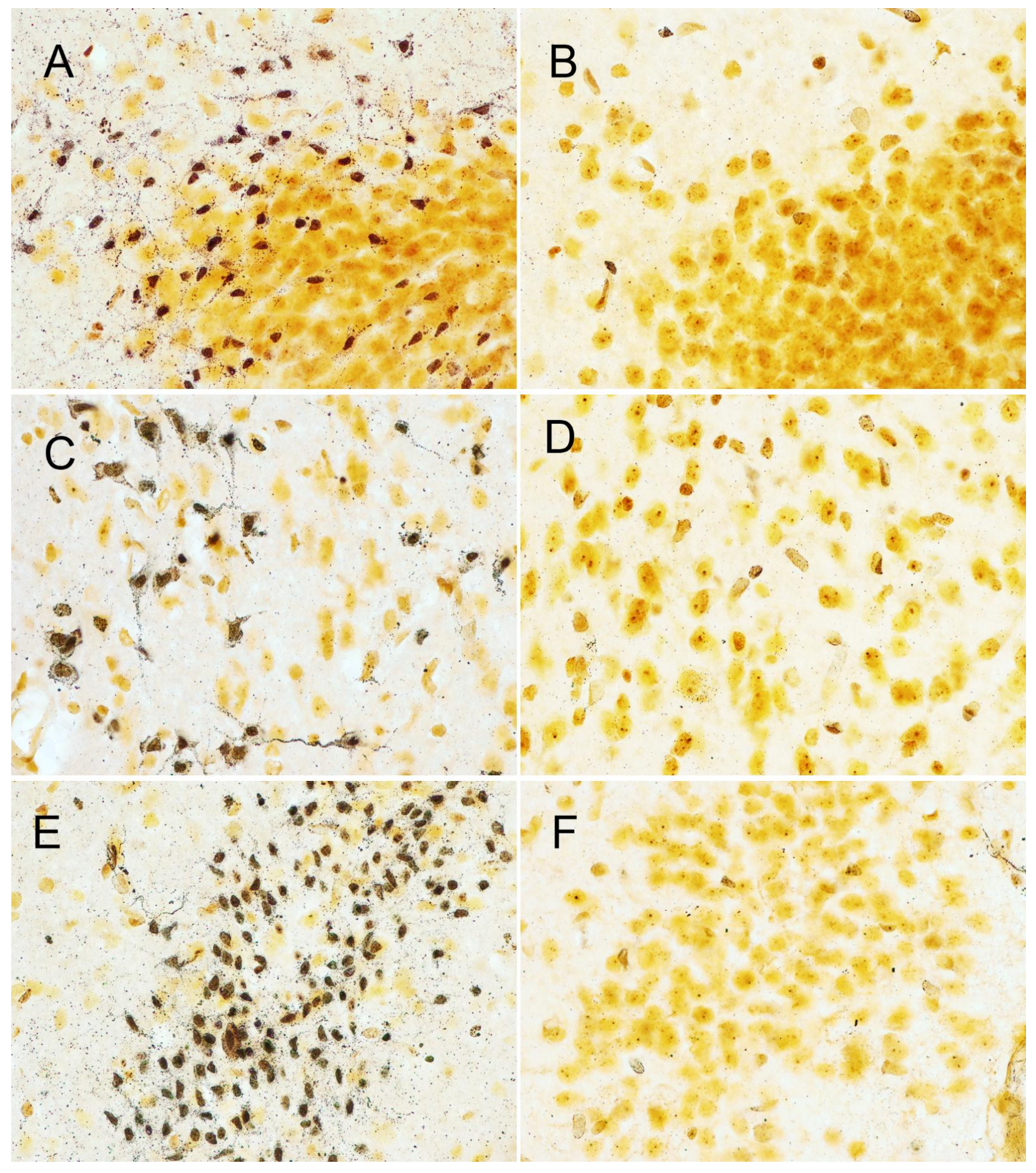




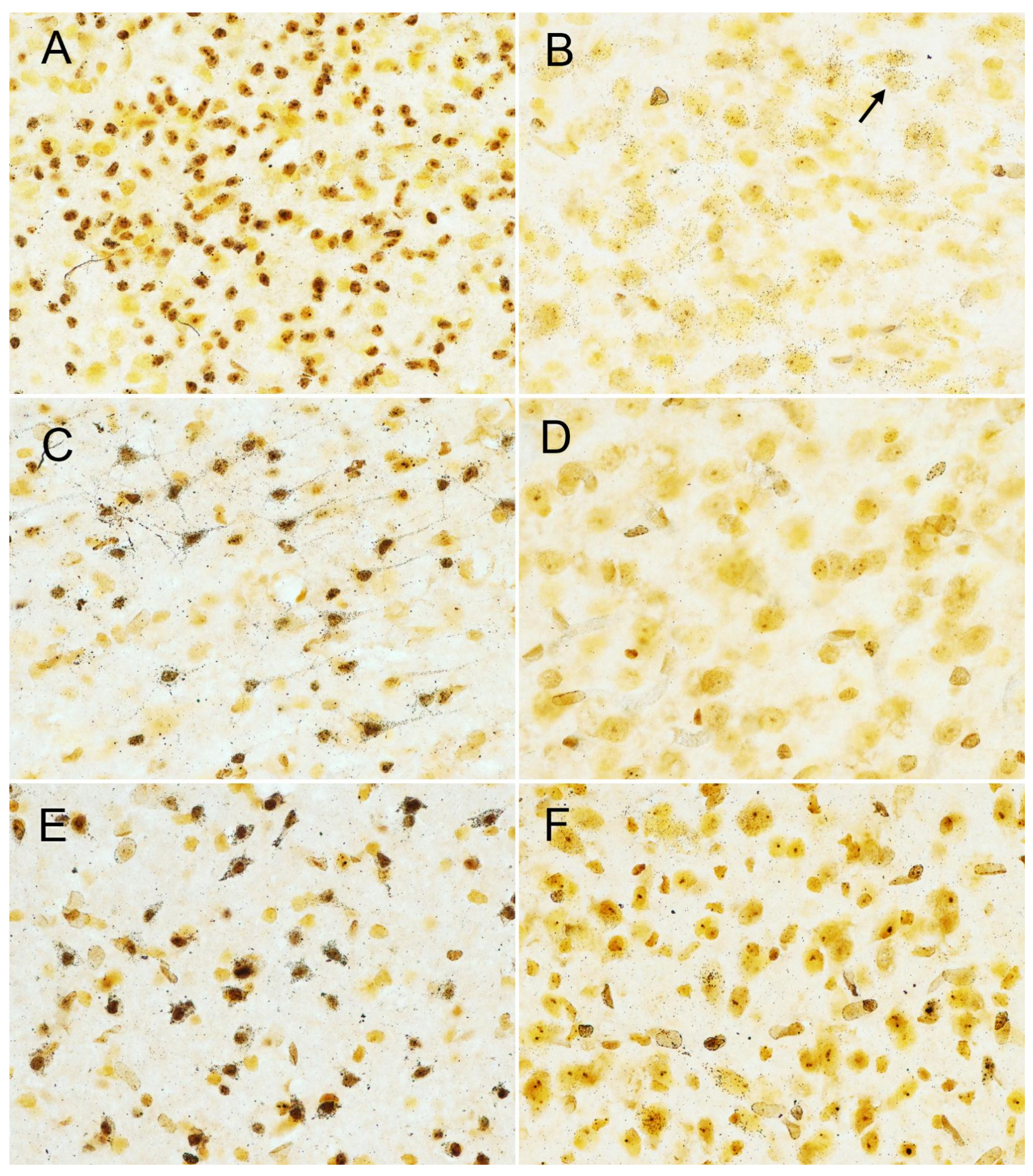




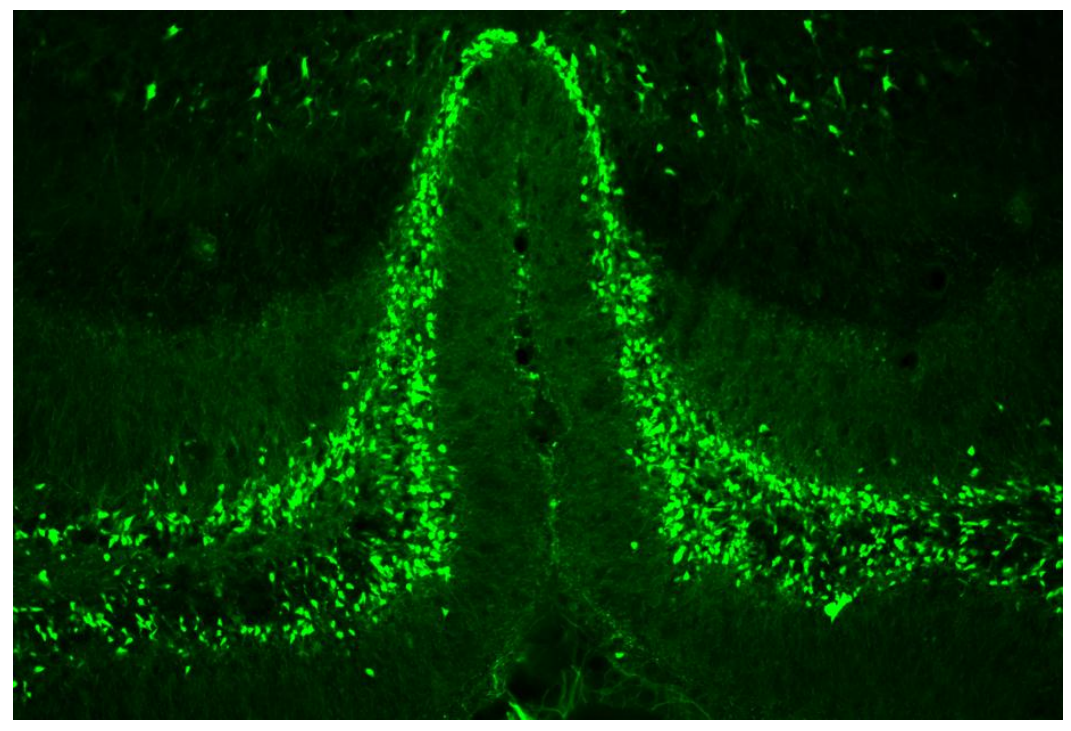


Intranasal administration of saline or obidoxime before or after paraoxon treatment

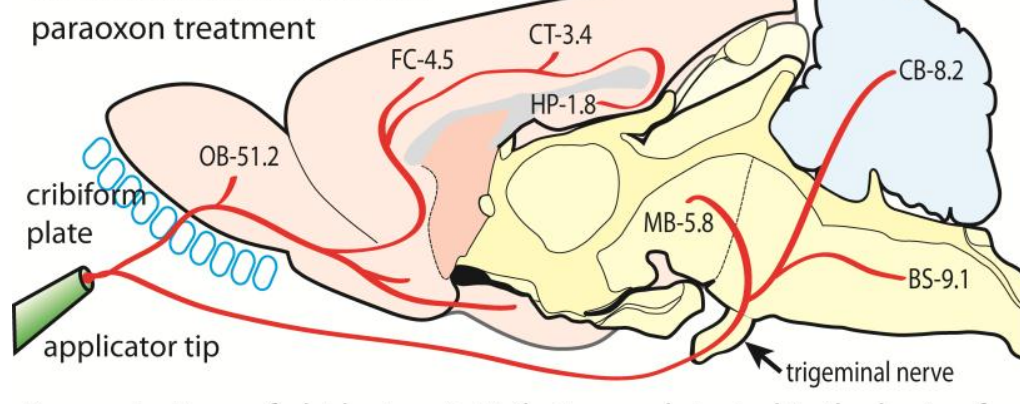

Saline

prolonged seizures

Fluoro-Jade B staining = neuronal loss

Concentrations of obidoxime $(\mu \mathrm{M})$ that were detected in the brain after Obidoxime passage through the olfactory and trigeminal pathways.

$\mathrm{OB}=$ olfactory bulb, $\mathrm{FC}=$ frontal cortex, $\mathrm{CT}=$ neocortex,

$\mathrm{HP}=$ hippocampus, $\mathrm{MB}=$ midbrain, $\mathrm{CB}=$ cerebellum and $\mathrm{BS}=$ brainstem 